\title{
LOCAL $\boldsymbol{H}$-MAPS OF CLASSIFYING SPACES
}

BY

\author{
TIMOTHY LANCE ${ }^{1}$
}

\begin{abstract}
Let $B U$ denote the localization at an odd prime $p$ of the classifying space for stable complex bundles, and let $f: B U \rightarrow B U$ be an $H$-map with fiber $F$. In this paper the Hopf algebra $H^{*}(F, Z / p)$ is computed for any such $f$. For certain $H$-maps $f$ of geometric interest the $p$-local cohomology of $F$ is given by means of the Bockstein spectral sequence. A direct description of $H^{*}\left(F, \mathbf{Z}_{(p)}\right)$ is also given for an important special case. Applications to the classifying spaces of surgery will appear later.
\end{abstract}

0. Introduction. This paper presents a computation of the cohomology of the fibers of maps $f: B U \rightarrow B U$, where $B U$ denotes the localization at a fixed odd prime $p$ of the classifying space for stable complex bundles, which preserve the $H$-multiplication induced by Whitney sum of bundles. Using the $p$-local $H$ space equivalence $B U \simeq B O \times \Omega^{2} B O$ a computation of the cohomology of the fibers of $H$-maps $B O \rightarrow B O$ is also obtained. In a subsequent paper these results will be applied to a detailed study of certain classifying spaces of surgery which arise in this fashion.

The viewpoint of this paper is motivated by a result of Adams that an $H$ map $f: B U \rightarrow B U$ is determined up to homotopy by the induced homomorphism $f_{\sharp}: \pi_{*}(B U) \rightarrow \pi_{*}(B U)$. If we write $\mathbf{Z}_{(p)}$ for the integers localized at $p$ and define the characteristic sequence $\lambda=\left(\lambda_{1}, \lambda_{2}, \ldots\right)$ of $f$ by the condition that $f_{\sharp}$ is multiplication by $\lambda_{j} \in \mathbf{Z}_{(p)}$ on $\pi_{2 j}(B U)=\mathbf{Z}_{(p)}$, then in principle the cohomology of the fiber can be completely described in terms of $\lambda$. We thus begin by studying the characteristic sequence, using as our main tool a natural decomposition of Hopf algebras $H^{*}\left(B U, \mathbf{Z}_{(p)}\right) \cong \bigotimes_{n \text { prime to } p} A_{n}^{*}$ in which $A_{n}^{*}$ is a polynomial Hopf algebra on generators $a_{n, k}^{*}$ of degree $2 n p^{k}$, $k=0,1, \ldots$

THEOREM A. If $f: B U \rightarrow B U$ is an $H$-map with characteristic sequence $\lambda$ and $n_{1} \equiv n_{2} \bmod (p-1)$, then $p^{j+1}$ divides $\lambda_{n_{1} p^{j}}-\lambda_{n_{2} p^{j}}$.

Define the surplus sequence $s(f)$ of $f$ by $s(f)_{m}=\nu\left(\lambda_{m}\right)-\nu(m)$ where $\nu(x)$ is the exponent of the highest power of $p$ dividing $x$. An important step in the

Received by the editors October 25, 1977 and, in revised form, July 18, 1978.

AMS (MOS) subject classifications (1970). Primary 55F20, 55H20, 55F45, $55 \mathrm{~J} 20$.

Key words and phrases. Localization, $\boldsymbol{H}$-map, torsion product, cohomology.

${ }^{1}$ Partially supported by NSF grant MCS7606740. 
proof of Theorem $\mathrm{A}$ is the fact that if $n$ is prime to $p$ and $j$ is minimal such that $s(f)_{n p^{j}} \leqslant 0$, then $s(f)_{n p^{j+k}}=-k$ for all $k \geqslant 0$. Thus for any $n$ prime to $p$ we may define $\delta_{n}=j$ if $s(f)_{n p^{j}}=0$ and $\delta_{n}=\infty$ if no such $j$ exists. For any integer $\delta \geqslant 0$ let $\xi^{\delta}$ denote the Frobenius map $x \rightarrow x^{p^{\delta}}$, and let $\xi^{\infty}$ be the augmentation.

Theorem B. Suppose $f: B U \rightarrow B U$ is an $H$-map with fiber $F$ and index $\delta_{n}$ defined as above. There is an isomorphism of Hopf algebras

$$
H^{*}(F, \mathbf{Z} / p) \cong \bigotimes_{n \text { prime top }} E\left\{\sigma a_{n, j}^{*} \mid 0 \leqslant j<\delta_{n}\right\} \otimes\left(A_{n}^{*} / / \xi^{\delta_{n}} A_{n}^{*} \otimes \mathbf{Z} / p\right)
$$

in which $\sigma: H^{q}(B U, \mathrm{Z} / p) \rightarrow H^{q-1}(F, \mathrm{Z} / p)$ is the cohomology suspension. Moreover, the map induced on cohomology by $F \rightarrow B U$ is given by the natural projections $A_{n}^{*} \otimes \mathrm{Z} / p \rightarrow A_{n}^{*} / / \xi^{\delta_{n}} A_{n}^{*} \otimes \mathrm{Z} / p$.

Perhaps the most transparent way to display the $p$-local cohomology is by means of the Bockstein spectral sequence. This is the graded $E_{1}$ spectral sequence associated to the exact couple given by the cohomology sequence of $0 \rightarrow \mathbf{Z} \rightarrow^{p} \mathbf{Z} \rightarrow \mathbf{Z} / p \rightarrow 0$. Of particular interest for geometric reasons is the Bockstein spectral sequence of the fiber of a "two-step function" $f$. This is an $H$-map such that $s(f)_{n}=s(f)_{p n}=\cdots=s(f)_{n p^{\delta_{n}-1}}=s_{n}>0$ for each $n$ prime to $p$ such that $\delta_{n}>0$.

THEOREM C. If $f: B U \rightarrow B U$ is a two-step function with fiber $F$, then the $E_{r}$ term of the Bockstein spectral sequence of $F$ is given by

$$
\begin{aligned}
& \bigotimes_{n \text { prime top }} E\left\{\sigma a_{n}^{*}, \overline{r-s_{n}}+j \mid 0 \leqslant j<\delta_{n}\right\} \\
& \otimes\left(A_{n}^{*}\left({\overline{r-s_{n}}}_{n}\right) / / \xi^{\delta_{n}} A_{n}^{*}\left({\overline{r-s_{n}}}_{n}\right) \otimes \mathbf{Z} / p\right)
\end{aligned}
$$

where $\overline{r-s_{n}}=\max \left\{0, r-s_{n}\right\}$ and

$$
A_{n}^{*}(k)=\mathbf{Z}_{(p)}\left[a_{n, k}^{*}, a_{n, k+1}^{*}, \ldots\right] \text { for any } k \geqslant 0 .
$$

In an application of this work to smoothing theory it will be necessary to have an explicit description of the $\mathbf{Z}_{(p)}$ cohomology of the fiber of an $H$-map $f: B U \rightarrow B U$ whose index $\delta_{n}$ never exceeds 1 . Let $S$ denote the set of all $n$ prime to $p$ such that $\delta_{n}=1$, and write $T$ for the set of all sequences $\alpha=\left(\alpha_{1}, \alpha_{2}, \ldots\right)$ of nonnegative integers each of whose nonzero entries is $\leqslant p-1$ and lies in one of the subsequences $\alpha_{n}, \alpha_{n p}, \ldots$ for some $n \in S$. For each $\alpha \in T$ write $l(\alpha)$ for the number of such subsequences containing a nonzero entry, and let $\operatorname{ind}(\alpha)=\min _{n \in S} p^{\nu\left(\lambda_{n}\right)+i_{n}}$ where $\alpha_{n p^{i_{n}}}$ is the first nonzero entry of $\alpha_{n}, \alpha_{p n}, \ldots$ (if none exists, $i_{n}=\infty$ ). Let $w(\alpha)=\alpha_{1}+2 \alpha_{2}+$ $3 \alpha_{3}+\ldots$ 
THEOREM D. If $f: B U \rightarrow B U$ is an $H$-map with fiber $F$ such that $\delta_{n} \leqslant 1$ for each $n$ prime to $p$, then for each $m>0$

$$
H^{m}\left(F, \mathbf{Z}_{(p)}\right) \cong \bigoplus_{j=1}^{\infty} \bigoplus_{i=0}^{\infty} \bigoplus_{\substack{\alpha \in T \\ l(\alpha)=j \\ 2 w(\alpha)=m+i}}(\mathbf{Z} / \operatorname{ind}(\alpha))^{\left(i_{i}^{-1}\right)}
$$

where the right-hand term denotes the direct sum of $\left({ }_{i}^{j-1}\right)$ copies of $Z / \operatorname{ind}(\alpha)$.

The paper is arranged as follows. In $\$ 1$ we discuss the arithmetic of characteristic sequences and the basic congruence of Theorem $\mathrm{A}$. This depends on the decomposition $H^{*}\left(B U, \mathbf{Z}_{(p)}\right) \cong \bigotimes_{n \text { prime to } p} A_{n}^{*}$ which is established in $\$ 2 . \$ 3$ is devoted to a detailed study of the homomorphism $f^{*}$ : $H^{*}\left(B U, \mathbf{Z}_{(p)}\right) \rightarrow H^{*}\left(B U, \mathbf{Z}_{(p)}\right)$ induced by an $H$-map $f$. This permits us to calculate torsion products for various $p$ local rings $R$ which, by some remarkable collapse theorems for the Eilenberg-Moore spectral sequence, is equivalent to computing the $R$ cohomology of the fiber of $f$. This is done for $R=\mathbf{Z} / p$ in $\S 4$ to obtain Theorem $\mathrm{B}$ and for $R=\mathbf{Z}_{(p)}$ in $\$ 5$ to obtain Theorem C. In $\$ 6$ we carry out the calculation Theorem D.

Finally, it is a pleasure to acknowledge the help of many people, especially J. P. May, R. Ming and T. MacGregor.

1. The characteristic sequence. All spaces in this paper are assumed to be localized at a fixed odd prime $p$ and thus have $p$-local homotopy groups and reduced homology groups ([25], [26]). Write $\mu$ for the localization at $p$ of the $H$-multiplication (or an appropriate iterate) induced by Whitney sum of bundles; $\mu$ corresponds to loop multiplication under the Bott equivalence $B U \simeq \Omega_{0}^{2} B U$. One immediate benefit of localizing is a geometric splitting of $B U$.

1.1 Theorem (AdAms-Peterson). There exist equivalences of infinite loop spaces

$$
\begin{aligned}
& B U \rightarrow W \times \Omega^{2} W \times \cdots \times{\Omega^{2 p-4} W}^{2} \text { and } \\
& B O \rightarrow W \times \Omega^{4} W \times \cdots \times \Omega^{2 p-6} W
\end{aligned}
$$

where $\pi_{2 k(p-1)}(W)=\mathbf{Z}_{(p)}, k=1,2, \ldots$, and $\pi_{i}(W)=0$ otherwise.

$W$ may be defined as the bottom space of a spectrum associated to a bordism theory with singularities [19]. The $H$-space decomposition can be found in [19] or [1], and the infinite loop equivalence then follows from [2]. Notice in particular that $B U \simeq B O \times \Omega^{2} B O$, so any $H$-map $f: B O \rightarrow B O$ may be regarded as a factor of the $H$-map $f \times 1: B U \rightarrow B U$ with the same fiber. Similarly, 1.1 and Bott periodicity yield an equivalence $B O \simeq B S p$.

For any map $f: B U \rightarrow B U$ we define a sequence $\lambda=\left(\lambda_{1}, \lambda_{2}, \ldots\right)$ in $\mathbf{Z}_{(p)}$ by the condition that $f_{\sharp}$ equals multiplication by $\lambda_{j}$ on $\pi_{2 j}(B U)=\mathbf{Z}_{(p)}$. If $f$ is an 
$H$-map (that is, if $f \mu=\mu(f \times f))$ we refer to $\lambda$ as the characteristic sequence of $f$ because of the following.

1.2 LEMMA [15, p. 100]. If two $H$-maps $f, g: B U \rightarrow B U$ have the same characteristic sequence they are homotopic.

Using 1.1 we may define infinite loop maps $\Omega^{2 i} W \rightarrow B U$ and $B U \rightarrow \Omega^{2 j} W$ such that the composite $\Omega^{2 i} W \rightarrow B U \rightarrow \Omega^{2 j} W$ is the identity or constant depending on whether or not $i=j$. Using these the following decomposition of $H$-maps follows directly from 1.2.

1.3 Corollary. For any $H$-map $f: B U \rightarrow B U$ let $\tilde{f}_{2 k}$ denote the composite $\Omega^{2 k} W \rightarrow B U \rightarrow^{f} B U \rightarrow \Omega^{2 k} W$. Then the diagram below homotopy commutes.

$$
\begin{array}{ccc}
B U & \stackrel{f}{\rightarrow} & B U \\
\simeq \downarrow & & \downarrow \simeq \\
\prod_{j=0}^{p-2} \Omega^{2 j} W & \tilde{f}_{0} \times \tilde{f}_{2} \times \cdots \times \tilde{f}_{2 p-4} & \prod_{j=0}^{p-2} \Omega^{2 j} W
\end{array}
$$

1.4 Operations on characteristic sequences. Given $H$-maps $f, g: B U \rightarrow B U$ with characteristic sequences $\lambda$ and $\eta$ we may form new $H$-maps $f+g=$ $\mu \circ(f \times g) \circ \Delta$ and $f \circ g$ whose characteristic sequences are the pointwise sum $\lambda+\eta$ and product $\lambda \cdot \eta$, respectively. The loop space inverse -1 has characteristic sequence $(-1,-1, \ldots)$, so that $-f=(-1) \circ f$ has sequence $-\lambda$. Adding copies of 1 or $(-1)$ we obtain maps with characteristic sequences of any constant integer value $n$. These are homotopy invertible for $n$ prime to $p$, and thus for any $\rho \in \mathbf{Z}_{(p)}$ there is an $H$-map with constant sequence $\rho$.

The splitting 1.3 induces a shearing operation. Let $S$ be some subset of $\{0,2, \ldots, 2 p-4\}$ and let

$$
\tilde{f}_{s}: \prod_{j=0}^{p-2} \Omega^{2 j} W \rightarrow \prod_{j=0}^{p-2} \Omega^{2 j} W
$$

be the product whose $2 j$ th component is $\tilde{f}_{2 j}$ if $2 j \in S$ and 1 otherwise. By 1.3, $\tilde{f}_{S}$ induces a unique $\boldsymbol{H}$-map $f_{S}: B U \rightarrow B U$ with characteristic sequence $\lambda(S)$ defined by $\lambda(S)_{n}=\lambda_{n}$ or 1 depending on whether or not $2 n+2 j \equiv 0$ $\bmod 2(p-1)$ for some $2 j \in S$. Setting $S=\{0,4, \ldots, 2 p-6\}$ we may thus identify an $H$-map $f: B O \rightarrow B O$ with $f_{S}: B U \rightarrow B U$ with characteristic sequence $\left(1, \lambda_{2}, 1, \lambda_{4}, \ldots\right)$ and $\operatorname{fiber}(f)=\operatorname{fiber}\left(f_{S}\right)$. We can also mix characteristic sequences. If $S_{1}$ and $S_{2}$ partition $\{0,2, \ldots, 2 p-4\}$, for example, then $f_{S_{1}} \circ g_{S_{2}}=g_{S_{2}} \circ f_{S_{1}}$ has characteristic sequence $\lambda\left(S_{1}\right) \cdot \eta\left(S_{2}\right)$, a mixture of $\lambda$ and $\eta$. Finally, note that by Bott periodicity we have a loop map $\Omega^{2 k} f$ : $B U \rightarrow B U$ whose characteristic sequence $\left(\lambda_{k+1}, \lambda_{k+2}, \ldots\right)$ is a left shift of $\lambda$. 
1.5 Definition. For any $\beta \in \mathbf{Z}_{(p)}$ let $\nu(\beta)$ denote the exponent of $p$ in a prime power decomposition of the numerator of $\beta$ (let $\nu(0)=\infty$ ). We say that $\beta_{1} \equiv \beta_{2} \bmod p^{k}$ if $\nu\left(\beta_{1}-\beta_{2}\right) \geqslant k$. Define the surplus sequence $s(f)$ of an $H$-map $f: B U \rightarrow B U$ with characteristic sequence $\lambda$ by $s(f)_{n}=\nu\left(\lambda_{n}\right)-\nu(n)$. A positive surplus sequence is necessary and sufficient for the triviality of $f^{*}$ : $H^{*}(B U, \mathrm{Z} / p) \rightarrow H^{*}(B U, \mathrm{Z} / p)$ (3.7), and in general surplus measures the torsion in the cohomology of the fiber (compare \$5). In \$3 we will also establish the following.

1.6 LeMma. For any $n$ prime to $p$, if $j$ is minimal such that $s(f)_{n p^{j}} \leqslant 0$, then $s(f)_{n p^{j+k}}=-k$.

1.7 TheOREM. Let $f: B U \rightarrow B U$ be an $H$-map with characteristic sequence $\lambda$. Then $\lambda_{m p^{k}} \equiv \lambda_{n p^{k}} \bmod p^{k+1}$ whenever $m \equiv n \bmod (p-1)$.

Proof. If we replace $f$ by $g=f-\lambda_{n p^{k}} \cdot 1$ as in 1.4, the resulting characteristic sequence has 0 as its $n p^{k}$ th term. In particular, $s(f)_{n p^{k}}>0$ and thus $s(f)_{n p^{j}}>0$ for $j<k$ by 1.6. But this means that $p^{j+1}$ divides $\lambda_{n p^{j}}-\lambda_{n p^{k}}$ for $j<k$. Using these congruences it thus suffices to check that $\lambda_{n p^{j}} \equiv \lambda_{(n+p-1) p^{j}}$ $\bmod p^{j+1}$ for any $n, j$. But if $k=p^{j}(n-1)$, it follows by 1.4 that $\Omega^{2 k} f$ has a characteristic sequence with $p^{j}$ th term $\lambda_{n p^{j}}$ and $p^{j+1}$ st term $\lambda_{(n+p-1) p^{j}}$. The desired congruence now follows from 1.6 as above.

1.8 RemarK. Suppose $f: B U \rightarrow B U$ is any map (not necessarily an $H$-map) with characteristic sequence $\lambda$. Then $\Omega^{2 j} f: B U \rightarrow B U$ is an $H$-map with characteristic sequence $\left(\lambda_{j+1}, \lambda_{j+2}, \ldots\right)$. Applying 1.7 for various values of $j$ it follows easily that $\lambda_{m p^{k}} \equiv \lambda_{n p^{k}} \bmod p^{k+1}$ if $m \equiv n \bmod (p-1)$ and $m \neq 1$ $\neq n$. I conjecture that the latter restriction on $m$ and $n$ is not necessary. But in any case, by the shearing construction of 1.4 we may identify any map $f$ : $B O \rightarrow B O$ with a map $f_{S}: B U \rightarrow B U$ with characteristic sequence $\left(1, \lambda_{2}, 1, \lambda_{4}, \ldots\right)$ so that 1.7 holds for $\lambda_{2}, \lambda_{4}, \ldots$ These congruences may be viewed as a generalized Kummer congruence; in a subsequent paper we study a cannibalistic class $\rho: B O \rightarrow B O^{\otimes}$ for which 1.7 reduces to the classical congruences between Bernoulli numbers.

2. Homology of local classifying spaces. Let $R$ be a commutative principal ideal domain which is $p$-local. This means that $R \cong R \otimes_{\mathbf{z}} \mathbf{Z}_{(p)}$ as a group or, equivalently, that multiplication by $m$ is bijective for $m$ prime to $p$. We write $c_{n} \in H^{2 n}(B U, R)$ for the class associated by localization to the $n$th Chern class, and note that cup product and the coproduct induced by the Whitney sum map $\mu$ give $H^{*}(B U, R)$ the following familiar Hopf algebra structure ([13], [16]). 
2.1 TheOREM. $H^{*}(B U, R)$ is a polynomial Hopf algebra $R\left[c_{1}, c_{2}, \ldots\right]$ with coproduct $\mu^{*} c_{n}=\sum c_{i} \otimes c_{n-i}$. If $d_{n} \in H_{2 n}(B U, R)$ is dual (in the basis of monomials) to $c_{1}^{n}$, then the correspondence $c_{n} \rightarrow d_{n}$ defines an isomorphism of Hopf algebras $H^{*}(B U, R) \rightarrow H_{*}(B U, R)$.

For any $n$-tuple $\alpha=\left(\alpha_{1}, \ldots, \alpha_{n}\right)$ of weight $w(\alpha)=\alpha_{1}+2 \alpha_{2}+\cdots+n \alpha_{n}$ write $c^{\alpha}$ for the cup product $c_{1}^{\alpha_{1}} \cdots c_{n}^{\alpha_{n}} \in H^{2 w(\alpha)}(B U, R)$ with dual class $d_{\alpha} \in H_{2 w(\alpha)}(B U, R)$, and let $d^{\alpha}=d_{1}^{\alpha_{1}} \cdots d_{n}^{\alpha_{n}}$ be the Pontrjagin product induced by $\mu_{*}$. Going full circle, write $c_{\alpha}$ for the dual of $d^{\alpha}$ in the monomial basis in $H_{*}(B U, R)$ (the Chern class $c_{n}$ is in fact dual to $\left.d_{1}^{n}\right)$. By 2.1 it follows that the primitives in $H^{*}(B U, R)$ and $H_{*}(B U, R)$ are generated as $R$ modules by $\left\{c_{e_{1}}, c_{e_{2}}, \ldots\right\}$ and $\left\{d_{e_{1}}, d_{e_{2}}, \ldots\right\}$, respectively (where $e_{n}=(0, \ldots, 0,1)$ is the $n$th unit vector), and $c_{e_{n}} \rightarrow d_{e_{n}}$ under the isomorphism of 1.2. By [5] we may choose generators $\omega_{n} \in \pi_{2 n}(B U)=\mathbf{Z}_{(p)}$ which are carried by the Hurewicz map to $(m-1) ! d_{e_{n}}$. We can also describe the primitives directly. For any $\alpha$ let $|\alpha|=\alpha_{1}+\alpha_{2}+\cdots+\alpha_{n}$ and

$$
\{\alpha\}=\left(\alpha_{1}+\cdots+\alpha_{n}\right) ! / \alpha_{1} ! \cdots \alpha_{n} !
$$

2.2 THEOREM. $d_{e_{n}}=\sum_{w(\alpha)=n}(-1)^{|\alpha|+n} n\{\alpha\} d^{\alpha} /|\alpha|$.

An identical formula holds for $c_{e_{n}}$. If we identify $c_{n}$ with the $n$th elementary symmetric function as in [4], then 2.2 is a consequence of Waring's formula for the Newton polynomials [13].

Given indeterminates $t_{0}, t_{1}, \ldots$ define the $k$ th Witt polynomial $T_{k}$ by $T_{k}(t)=t_{0}^{p^{k}}+p t_{1}^{p^{k-1}}+\cdots+p^{k} t_{k}$ where we abbreviate $t=\left(t_{0}, t_{1}, \ldots\right)$. Let $t^{p}=\left(t_{0}^{p}, t_{1}^{p}, \ldots\right)$.

2.3 LemMA. Let $P_{0}, P_{1}, \ldots$ be polynomials in $t_{0}, t_{1}, \ldots$ with coefficients in $R$ (respectively, Z) such that $P_{k}(t)-P_{k-1}\left(t^{p}\right)$ vanishes $\bmod p^{k}$. Then the equations $P_{k}(t)=T_{k}\left(\varphi_{0}(t), \varphi_{1}(t), \ldots, \varphi_{k}(t)\right)$ inductively define polynomials $\varphi_{0}, \varphi_{1}, \ldots$ with coefficients in $R$ (respectively, $\mathbf{Z}$ ).

Proof. Suppose the polynomials $\boldsymbol{P}_{i}$ are integral, the proof for $\boldsymbol{R}$ being identical. Since $\varphi_{0}=P_{0}$, we assume inductively that $\varphi_{0}, \ldots, \varphi_{k-1}$ are integral for some $k>0$. Since $T_{k}(t)=T_{k-1}\left(t^{p}\right)+p^{k} t_{k}$, we must verify that $P_{k}(t)-$ $T_{k-1}\left(\left(\varphi_{0}(t)\right)^{p}, \ldots,\left(\varphi_{k-1}(t)\right)^{p}\right)$ vanishes $\bmod p^{k}$.

Evidently the integral polynomials $\left(\varphi_{j}(t)\right)^{p}$ and $\varphi_{j}\left(t^{p}\right)$ are congruent $\bmod p$. Applying the binomial theorem inductively, it follows that $\left(\varphi_{j}(t)\right)^{p^{j+1}}$ and $\varphi_{j}\left(t^{p}\right)^{p^{j}}$ are congruent $\bmod p^{j+1}$. Consequently,

$$
T_{k-1}\left(\left(\varphi_{0}(t)\right)^{p}, \ldots,\left(\varphi_{k-1}(t)\right)^{p}\right) \equiv T_{k-1}\left(\varphi_{0}\left(t^{p}\right), \ldots, \varphi_{k-1}\left(t^{p}\right)\right) \bmod p^{k}
$$

But $P_{k-1}(t)=T_{k-1}\left(\varphi_{0}(t), \ldots, \varphi_{k-1}(t)\right)$ by definition, and so $P_{k-1}\left(t^{p}\right)=$ $T_{k-1}\left(\varphi_{0}\left(t^{p}\right), \ldots, \varphi_{k-1}\left(t^{p}\right)\right)$. The lemma now follows from the congruence $P_{k}(t) \equiv P_{k-1}\left(t^{p}\right) \bmod p^{k}$. 
2.4 Corollary. For each $n$ prime to $p$ and $k \geqslant 0$ there exists $a_{n, k} \in$ $H_{2 n p^{k}}(B U, R)$ defined inductively by $d_{e_{n p^{k}}}=T_{k}\left(a_{n, 0}, \ldots, a_{n, k}\right)$.

Proof. Let $S_{n}$ denote the polynomial of 2.2. By 2.3 we must show that

$$
S_{n p^{k}}\left(d_{1}, \ldots, d_{n p^{k}}\right)-S_{n p^{k-1}}\left(d_{1}, \ldots, d_{n p^{k-1}}\right)
$$

vanishes $\bmod p^{k}$. For any $\alpha$ of weight $w(\alpha)=n p^{k}$ with some entry prime to $p$, the coefficient of $d^{\alpha}$ in (*) is $n p^{k}\{\alpha\} /|\alpha|$. We must check that $\nu(\{\alpha\})>$ $\nu(|\alpha|)$ where $\nu(\alpha)$ is the exponent of $p$ in a prime power decomposition. But $\{\alpha\}=\left\{\alpha_{1}, \alpha_{2}+\cdots+\alpha_{k}\right\} \cdot\left\{\alpha_{2}, \ldots, \alpha_{k}\right\}$, so this follows from the familiar $\nu(\{\beta\}) \geqslant \nu(|\beta|)$ where $\beta=\left(\beta_{1}, \beta_{2}\right)$ with $\beta_{1}$ prime to $p$.

In fact, the inequality $\nu\left(\left\{p^{m} \beta\right\}\right) \geqslant \nu(|\beta|)$ holds for any $\beta=\left(\beta_{1}, \ldots, \beta_{s}\right)$. As before, it suffices to show this for $s=2$ with $\beta_{1}$ prime to $p$. Let $\pi(i)$ denote the product of all natural numbers $<i$ which are prime to $p$. By some simple bookkeeping we obtain $\{p \beta\} /\{\beta\}=\pi\left(p \beta_{1}+p \beta_{2}\right) / \pi\left(p \beta_{1}\right) \pi\left(p \beta_{2}\right)$ and hence

$$
\left\{p^{m} \beta\right\} \prod_{i=1}^{m} \pi\left(p^{i} \beta_{1}\right) \pi\left(p^{i} \beta_{2}\right)=\{\beta\} \prod_{i=1}^{m} \pi\left(p^{i}\left(\beta_{1}+\beta_{2}\right)\right) .
$$

But $\nu(\{\beta\}) \geqslant \nu(|\beta|)$ from above, while $\nu\left(\left\{p^{m} \beta\right\}\right)=\nu(\{\beta\})$ since $\pi$ takes values prime to $p$.

Returning to the polynomial (*), if $\alpha=p \beta$ then by 2.2 the coefficient of $d^{\alpha}$ is

$$
\pm\left(\frac{n p^{k}}{|p \beta|}\{p \beta\}-\frac{n p^{k-1}}{|\beta|}\{\beta\}\right)= \pm \frac{n p^{k-1}}{|\beta|}(\{p \beta\}-\{\beta\})
$$

But since $\{p \beta\} /\{\beta\}=\pi(|p \beta|) / \pi\left(p \beta_{1}\right) \cdots \pi\left(p \beta_{n p}\right)$ it follows that

$$
\begin{aligned}
\pi\left(p \beta_{1}\right) \cdots \pi\left(p \beta_{n p^{k}}\right) & (\{p \beta\}-\{\beta\}) \\
= & \{\beta\}\left(\pi(|p \beta|)-\pi\left(p \beta_{1}\right) \cdots \pi\left(p \beta_{n p^{k}}\right)\right) .
\end{aligned}
$$

If $q=\min \left(\nu\left(\beta_{1}\right), \ldots, \nu\left(\beta_{n p^{k}}\right)\right)$, then $\nu(\{\beta\}) \geqslant \nu(|\beta|)-q$ by the paragraph above. By Wilson's Theorem (see e.g. [20]), $\pi(|p \beta|) \equiv(-1)^{|\beta| / p^{q}} \bmod p^{q+1}$ and $\pi\left(p \beta_{i}\right) \equiv(-1)^{\left|\beta_{i}\right| / p^{q}} \bmod p^{q+1}$. Thus $\pi(|p \beta|)-\pi\left(p \beta_{1}\right) \cdots \pi\left(p \beta_{n p^{k}}\right) \equiv 0$ $\bmod p^{q+1}$ so that $p^{p(|\beta|)+1}$ divides $\{p \beta\}-\{\beta\}$, and the coefficients of $d^{\alpha}$ in (*) vanishes $\bmod p^{k}$.

Using 2.2 it follows that $a_{n, k}=n d_{n p^{k}}+$ decomposables and thus $\left\{a_{n, k} \mid n\right.$ is prime to $p, k \geqslant 0\}$ is a polynomial basis of $H_{*}(B U, R)$. For each $n$ prime to $p$ let $A_{n}$ denote the subalgebra of $H_{*}(B U, R)$ generated by $a_{n, 0}, a_{n, 1}, \ldots$ Since $A_{n}$ is pure in $H_{*}\left(B U, \mathbf{Z}_{(p)}\right)$ (if $m x \in A_{n}$ for some integer $m$ then $x \in A_{n}$ ) and $d_{e_{n k}}$ is primitive, it follows from the defining relation for $a_{n, k}$ that $A_{n}$ is a Hopf subalgebra for $R=\mathbf{Z}_{(p)}$ and hence for any $p$-local $R$ by universal coefficients. This proves the first part of the following result which was first established by Husemoller using different methods [11]. 
2.5 THEOREM. There are isomorphisms of Hopf algebras

$$
H_{*}(B U, R) \cong \bigotimes_{*}\left(\Omega^{2 k} W, R\right) \cong \underbrace{\bigotimes}_{\substack{n \text { prime top } p \\ n+k \equiv 0 \bmod (p-1)}} A_{n} \text { and }
$$

When $R=\mathbf{Z}_{(p)}$ this decomposition is best possible: given Hopf subalgebras $B, C \subseteq H_{*}\left(B U, \mathbf{Z}_{(p)}\right)$ such that $H_{*}\left(B U, \mathbf{Z}_{(p)}\right)=B \otimes C$, then for any $n$ prime to $p$ either $A_{n} \subseteq B$ or $A_{n} \subseteq C$.

Proof. We prove the last statement first. Since $B$ and $C$ are pure, it follows from $[17,6.16]$ that any primitive $d_{e_{n, j}}$ is in $B$ or in $C$. Suppose inductively that $d_{e_{n}}=a_{n, 0}, a_{n, 1}, \ldots, a_{n, j-1} \in B$. By $2.4, x=d_{e_{n j}}-T_{j}\left(\left(a_{n, 0}\right)^{p}, \ldots,\left(a_{n, j-1}\right)^{p}\right)$ is $p$-divisible and thus so is its image $[x]$ in $H_{*}\left(B U, \mathbf{Z}_{(p)}\right) / / B \cong C$. But if $d_{e_{n j}} \in C,[x]=d_{e_{n j}}$ which is not $p$-divisible by 2.2. Thus $d_{e_{n j}}$ and hence $a_{n, j}$ and $A_{n}$ lie in $B$.

Because the Hurewicz map sends some generator $\omega_{m} \in \pi_{2 m}(B U)=\mathbf{Z}_{(p)}$ to $(m-1) ! d_{e_{m}}$, it follows from 1.1 that $H_{*}\left(\Omega^{2 k} W, \mathbf{Z}_{(p)}\right)$ is the smallest pure Hopf subalgebra of $H_{*}\left(B U, \mathbf{Z}_{(p)}\right)$ containing the primitives $d_{e_{n j}}$ where $n+k \equiv 0$ $\bmod (p-1)$. Then from the argument above we have

$$
H_{*}\left(\Omega^{2 k} W, R\right)=\bigotimes_{\substack{n \text { prime to } p \\ n+k \equiv 0 \bmod (p-1)}}^{\bigotimes} A_{n}
$$

for $R=\mathbf{Z}_{(p)}$ and hence for any $p$-local $R$ by universal coefficients.

By the same arguments we have a decomposition in cohomology.

2.6 TheOREM. For $n$ prime to $p$ and $j \geqslant 0$ define $a_{n j}^{*} \in H^{2 n p^{j}}(B U, R)$ inductively by $c_{e_{n j}}=T_{k}\left(a_{n, 0}^{*}, \ldots, a_{n, j}^{*}\right)$. Let $A_{n}^{*}$ denote the polynomial Hopf subalgebra $R\left[a_{n, 0}^{*}, a_{n, 1}^{*}, \ldots\right]$. Then there exist isomorphisms of Hopf algebras

$$
\begin{aligned}
& H^{*}(B U, R) \cong \bigotimes_{n \text { prime to } p} A_{n}^{*} \text { and }
\end{aligned}
$$

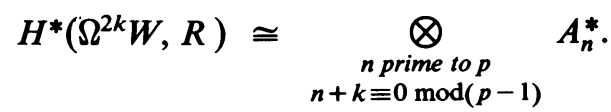

The homology and cohomology decompositions of $B U$ (and of $\Omega^{2 k} W$ ) are dual. If we give $H_{*}(B U, R)$ the basis of monomials in $\left\{a_{n, j}\right\}$, then $c_{e_{n p^{k}}} / n$ is dual to $a_{n, k}$. The subalgebras $A_{n}$ and $A_{n}^{*}$ are in some sense the minimal bipolynomial Hopf algebras [21].

3. The homology of $H$-maps. We can now describe the induced homomorphism of an $H$-map $f: B U \rightarrow B U$. Since the Hurewicz map induces an isomorphism between $\pi_{*}(B U) \otimes Q$ and the primitives of $H_{*}(B U, Q)$ ([5], [17]), the characteristic sequence $\lambda$ of $f$ may be defined by $f_{*}\left(d_{e_{m}}\right)=\lambda_{m} d_{e_{m}}$ 
where $d_{e_{m}}$ is as in 2.2. Thus for each $n$ prime to $p$ and $k \geqslant 0$ we may apply $f_{*}$ to the defining relation 2.4 to obtain

$$
\lambda_{n p^{k}} T_{k}\left(a_{n, 0}, \ldots, a_{n, k}\right)=T_{k}\left(f_{*}\left(a_{n, 0}\right), \ldots, f_{*}\left(a_{n, k}\right)\right) .
$$

We illustrate the use of this equation in the ring $H_{*}\left(B U, \mathbf{Z}_{(p)}\right)$ where the $a_{n, k}$ are not $p$-divisible. Suppose $\nu\left(\lambda_{n p^{k}}\right)=j>0$. Then $\left(f_{*}\left(a_{n, 0}\right)\right)^{p^{k}}$ is divisible by $p$ and hence by $p^{p^{k}}$. If $j>1$ divides both sides of 3.1 by $p$, conclude that $\left(f_{*}\left(a_{n, 1}\right)\right)^{p^{k-1}}$ is divisible by $p^{p^{k-1}}$, and continue inductively. It follows that $f_{*}\left(a_{n, 0}\right), \ldots, f_{*}\left(a_{n, \min (j-1, k)}\right)$ all vanish $\bmod p$ and, if $(j-1)<k$, that $f_{*}\left(a_{n, j}\right)$ $\neq 0$.

Restating this in terms of surplus (1.5), we have that $s(f)_{n p^{k}}>0$ iff $f_{*}\left(a_{n, 0}\right), \ldots, f_{*}\left(a_{n, k}\right)$ all vanish $\bmod p$. Moreover, if $s(f)_{n p^{k}} \leqslant 0$ and $j=k+$ $s\left(f_{n p^{k}}\right.$, then $f_{*}\left(a_{n, 0}\right), \ldots, f_{*}\left(a_{n, j-1}\right)$ all vanish $\bmod p$ while $f_{*}\left(a_{n, j}\right)$ does not. In particular, if $f_{*}\left(a_{n, k}\right)$ is the first nonvanishing $(\bmod p)$ term of $f_{*}\left(a_{n, 0}\right)$, $f_{*}\left(a_{n, 1}\right), \ldots$, then we must have $s(f)_{n p^{k}}=0, s(f)_{n p^{k+1}}=-1, \ldots$ This proves Assertion 1.6.

3.2 Definition. For any $H$-map $f: B U \rightarrow B U$ and $n$ prime to $p$ define the $n$th index of $f$, denoted $\delta_{n}(f)=\delta_{n}$, by $\delta_{n}=j$ if $s(f)_{n p^{j}}=0$ and $\delta_{n}=\infty$ if no such $j$ exists.

By 1.7 the index $\delta_{n}$ is constant as $n$ varies within a residue class $\bmod (p-$ 1). The discussion above may be summarized by saying that $f_{*}\left(a_{n, j}\right)$ vanishes $\bmod p$ for $j<\delta_{n}$ while $f_{*}\left(a_{n, \delta_{n}}\right) \not \neq 0 \bmod p$.

\subsection{THEOREM. For each $n$ prime to $p$ and $k \leqslant \delta_{n}$ we have}

$$
f_{*}\left(a_{n, k}\right)=\left(\lambda_{n p^{k}} / p^{k}\right) T_{k}\left(a_{n, 0}, \ldots, a_{n, k}\right)+p x
$$

for some $x$ in the ideal in $A_{n}$ generated by all $f_{*}\left(a_{n, i}\right), i<k$. If $f$ satisfies the growth condition $s(f)_{n p^{i}}<p s(f)_{n p^{i-1}}-1$ for $i \leqslant k$, then $\nu(p x)>\nu\left(\lambda_{n p^{k}} / p^{k}\right)=$ $s(f)_{n p^{k}}$ and thus $\nu\left(f_{*}\left(a_{n, k}\right)\right)=s(f)_{n p^{k}}$.

For the final statement we assume that the ground ring $R$ is $\mathbf{Z}_{(p)}$. For $x \in H_{*}(B U, R)$ the symbol $\nu(x)$ then denotes the maximal $j$ such that $x=p^{j} y$ for some $y \in H_{*}(B U, R)$. The growth condition is not satisfied in general (examples can be easily constructed using 1.4) but is satisfied by a number of maps $f$ of geometric interest. The last statement often permits an explicit description of the torsion in the $\mathbf{Z}_{(p)}$ cohomology of the fiber of $f$.

Proof. If we solve the defining equation 3.1 for $f_{*}\left(a_{n, k}\right)$ we obtain

$$
\left(\lambda_{n p^{k}} / p^{k}\right) T_{k}\left(a_{n, 0}, \ldots, a_{n, k}\right)-\left(1 / p^{k}\right) T_{k-1}\left(\left(f_{*}\left(a_{n, 0}\right)\right)^{p}, \ldots,\left(f_{*}\left(a_{n, k-1}\right)\right)^{p}\right) .
$$

But $\left(f_{*}\left(a_{n, i}\right)\right)^{p^{k-i}} / p^{k-i}=\left(\left(f_{*}\left(a_{n, i}\right)\right)^{p^{k-i}-1} / p^{k-i}\right) f_{*}\left(a_{n, i}\right)$ when $i<k$ where the first factor is evidently $p$-divisible since $f_{*}\left(a_{n, i}\right)$ is. Thus the second polynomial above has the desired form $p x$. 
To prove the second assertion we may suppose inductively that $\nu\left(f_{*}\left(a_{n, i}\right)\right)$ $=s(f)_{n p}$ for $i<k$ and thus

$$
\nu\left(\left(f_{*}\left(a_{n, i}\right)\right)^{p^{k-i}} / p^{k-i}\right)=p^{k-i} s(f)_{n p^{i}}-(k-i) .
$$

But the growth condition implies that $p s(f)_{n p^{k-1}}-1<p^{2} s(f)_{n p^{k-2}}-2<\ldots$ and hence

$$
\nu(p x)=\nu\left(\left(f_{*}\left(a_{n, k-1}\right)\right)^{p} / p\right)=p s(f)_{n p^{k-1}}-1>s(f)_{n p^{k}}
$$

Evaluating $f_{*}\left(a_{n, k}\right)$ for $k>\delta_{n}$ is more difficult since $\lambda_{n p^{k}}$ and $f_{*}\left(a_{n, i}\right)$ have insufficient $p$-divisibility to carry out the manipulations above. A less direct approach seems more fruitful. Fix some $n$ prime to $p$ with finite $\delta_{n}$ and for each $k>\delta_{n}$ let $u_{k}=\lambda_{n p^{k}} / p^{\delta_{n}}$, a unit in $\mathbf{Z}_{(p)}$. By 1.7 it follows that $\left(1 / u_{k}\right) \equiv$ $\left(1 / u_{k-1}\right) \bmod p^{k-\delta_{n}}$. Then using 2.3 we may inductively define polynomials $\rho_{k-\delta_{n}}(t)=\rho_{k-\delta_{n}}\left(t_{0}, \ldots, t_{k-\delta_{n}}\right)$ and $\xi_{k-\delta_{n}}(t)=\xi_{k-\delta_{n}}\left(t_{1}, \ldots, t_{k}\right)$ for $k>\delta_{n}$ as follows.

$$
\begin{gathered}
\left(1 / u_{k}\right) T_{k-\delta_{n}}(t)=T_{k-\delta_{n}}\left(\rho_{0}(t), \ldots, \rho_{k-\delta_{n}}(t)\right), \\
-p^{k-\delta_{n}+1} T_{\delta_{n}-1}\left(t_{k-\delta_{n}+1}, \ldots, t_{k}\right)=T_{k-\delta_{n}}\left(\xi_{0}(t), \ldots, \xi_{k-\delta_{n}}(t)\right) .
\end{gathered}
$$

It follows immediately that $\xi_{k-\delta_{n}}$ is always $p$-divisible and that $\rho_{k-\delta_{n}}(t)=$ $\left(1 / u_{k}\right) t_{k}+$ decomposables. Hence the following result includes a partial description of $f_{*}\left(a_{n, k}\right)$.

3.5 THEOREM. If $\boldsymbol{n}$ is prime to $\boldsymbol{p}$ and $k \geqslant \delta_{\boldsymbol{n}}$ then

$$
a_{n, k-\delta_{n}}^{p^{\delta_{n}}}=\rho_{k-\delta_{n}}\left(f_{*}\left(a_{n, \delta_{n}}\right), \ldots, f_{*}\left(a_{n, k}\right)\right)+\xi_{k-\delta_{n}}\left(a_{n, 1}, \ldots, a_{n, k}\right)+p x
$$

for some $x$ in the ideal in $A_{n}$ generated by all $f_{*}\left(a_{n, i}\right), i<k$.

Proof. For the proof we abbreviate $a_{n, i}=a_{i}, \xi_{i-\delta_{n}}\left(a_{1}, \ldots, a_{i}\right)=\xi_{i-\delta_{n}}$, and $\rho_{i-\delta_{n}}\left(f_{*}\left(a_{\delta_{n}}\right), \ldots, f_{*}\left(a_{i}\right)\right)=\rho_{i-\delta_{n}}$. For $k=\delta_{n}$ the result is established in 3.3, so suppose inductively that 3.5 holds for all $i$ such that $\delta_{n}<i<k$. Then using the relations $a_{i}^{p_{n}}=\rho_{i-\delta_{n}}+\xi_{i-\delta_{n}}+p x_{i}$, the $p$-divisibility of $\xi_{i-\delta_{n}}$, and the multinomial theorem it follows (compare the proof of 2.4) that

$$
\begin{aligned}
T_{k-\delta_{n}-1}\left(a_{0}^{\delta_{n}+1}, \ldots, a_{k-\delta_{n}-1}^{\delta_{n}+1}\right)= & T_{k-\delta_{n}-1}\left(\rho_{0}^{p}, \ldots, \rho_{k-\delta_{n}-1}^{p}\right) \\
& +T_{k-\delta_{n}-1}\left(\xi_{0}^{p}, \ldots, \xi_{k-\delta_{n}-1}\right)+p^{k-\delta_{n}+1} \bar{x}
\end{aligned}
$$

for some $\bar{x}$ in the ideal generated by $f_{*}\left(a_{0}\right), \ldots, f_{*}\left(a_{k-1}\right)$. If we multiply the defining equations 3.1 by the unit $p^{\delta_{n}} / \lambda_{k}=1 / u_{k}$ and substitute the identities $(*)$, the left-hand side of the resulting equation is

$$
\begin{aligned}
p^{\delta_{n}} T_{k-\delta_{n}-1}\left(\rho^{p}\right)+p^{\delta_{n}} T_{k-\delta_{n}-1}\left(\xi^{p}\right)+p^{k+1} \bar{x} & \\
& +p^{k} a a_{-\delta_{n}}^{\delta_{n}}+p^{k+1} T_{\delta_{n}-1}\left(a_{k-\delta_{n}+1}, \ldots, a_{k}\right) .
\end{aligned}
$$


Substituting the defining relation 3.4 for $\xi_{k-\delta_{n}}$ and simplifying via the identity $T_{k-\delta_{n}}(\xi)=T_{k-\delta_{n}-1}\left(\xi^{p}\right)+p^{k-\delta_{n}} \xi_{k-\delta_{n}}$ this becomes

$$
p^{\delta_{n}} T_{k-\delta_{n}-1}\left(\rho^{p}\right)+p^{k+1} \bar{x}+p^{k} a_{k-\delta_{n}}^{p_{n}}-p^{k} \xi_{k-\delta_{n}}
$$

Using the arguments of 3.3 it follows that the right-hand side of 3.1 (multiplied by $p^{\delta_{n}} / \lambda_{n p^{k}}=1 / u_{k}$ as before) may be written as

$$
p^{k+1} \overline{\bar{x}}+\left(p^{\delta_{n}} / u_{n}\right) T_{k-\delta_{n}}\left(f_{*}\left(a_{\delta_{n}}\right), \ldots, f_{*}\left(a_{k}\right)\right)
$$

for some $\overline{\bar{x}}$ in the ideal generated by $f_{*}\left(a_{0}\right), \ldots, f_{*}\left(a_{k-1}\right)$. Then substituting the defining equation 3.4 for $\rho_{k-\delta_{n}}$ and the identity $T_{k-\delta_{n}}(\rho)=T_{k-\delta_{n}-1}\left(\rho^{p}\right)+$ $p^{k-\delta_{n}} \rho_{k-\delta_{n}}$ this becomes

$$
p^{k+1} \overline{\bar{x}}+p^{\delta_{n}} T_{k-\delta_{n}-1}\left(\rho^{p}\right)+p^{k} \rho_{k-\delta_{n}}
$$

The desired result follows by equating (**) and (***).

The above work made no use of the properties of the $H$-map $f$ beyond the relation 3.1. Since the cohomology homomorphism $f^{*}: H^{*}(B U, R) \rightarrow$ $H^{*}(B U, R)$ sends each primitive $c_{e_{n}}$ to some multiple of itself and since $\left\langle c_{e_{n}}, d_{e_{n}}\right\rangle= \pm n$, it follows that $f^{*}\left(c_{e_{n}}\right)=\lambda_{n} c_{e_{n}}$ and 3.3 and 3.5 hold for cohomology.

3.6 THEOREM. For each $n$ prime to $p$ we have

$$
\begin{aligned}
& f^{*}\left(a_{n, k}^{*}\right)=\left(\lambda_{n p^{k}} / p^{k}\right) T_{k}\left(a_{n, 0}^{*}, \ldots, a_{n, k}^{*}\right)+p x_{1}^{*} \quad \text { for } k<\delta_{n} \text {, } \\
& \left(a_{n, k-\delta_{n}}^{*}\right)^{p^{\delta_{n}}}=\rho_{k-\delta_{n}}\left(f^{*}\left(a_{n, \delta_{n}}^{*}\right), \ldots, f^{*}\left(a_{n, k}\right)\right)+\xi_{k-\delta_{n}}\left(a_{n, 1}^{*}, \ldots, a_{n, k}^{*}\right)+p x_{2}^{*} \\
& \text { if } k \geqslant \delta_{n}
\end{aligned}
$$

where $x_{j}^{*}$ lies in the ideal in $A_{n}^{*}$ generated by all $f^{*}\left(a_{n, i}^{*}\right), i<k$, and $\xi_{k-\delta_{n}}, \rho_{k-\delta_{n}}$ are as in 3.4. If $f$ satisfies the additional growth condition $s(f)_{n p^{i}}<p s(f)_{n p^{i-1}}-$ 1 for $i<\delta_{n}$, then $\nu\left(f^{*}\left(a_{n, k}^{*}\right)\right)=s(f)_{n p^{k}}$ for $k \leqslant \delta_{n}$.

For the last statement we again assume that the ground ring is $\mathbf{Z}_{(p)}$. The above result (and the homology analogue) simplifies considerably if we reduce $\bmod p$.

3.7 Corollary. The Hopf algebra homomorphism $f^{*}: H^{*}(B U, \mathrm{Z} / p) \rightarrow$ $H^{*}(B U, \mathrm{Z} / p)$ induced by an $H$-map $f$ satisfies

$$
f^{*}\left(a_{n, k}^{*}\right)= \begin{cases}0 & \text { if } k<\delta_{n}, \\ u_{k}\left(a_{n, k-\delta_{n}}^{*}\right)^{p^{\delta_{n}}}+x_{k} & \text { if } k>\delta_{n},\end{cases}
$$

where $x_{k}$ lies in the ideal in $A_{n}^{*}$ generated by all $\left(a_{n, j}^{*}\right)^{p^{\delta_{n}}}, j<k-\delta_{n}-1$, and $u_{k} \in \mathbf{Z}_{(p)}$ is a unit. 
4. The $\bmod p$ cohomology of the fiber. For any commutative Noetherian ring $R$ and for any simple fibration $F \rightarrow E \rightarrow B$ with $E$ and $B$ connected and of finite type over $R$ there is an Eilenberg-Moore spectral sequence $\left\{E_{r}\right\}$ converging from $E_{2}=\operatorname{Tor}_{H^{*}(B, R)}\left(R, H^{*}(E, R)\right)$ to $E_{\infty}=E_{0} H^{*}(F, R)$, the bigraded module associated to some filtration of $H^{*}(F, R)$ ([9], [10]). This spectral sequence is natural for morphisms of fibrations, and the filtration on $H^{*}(F, R)$ behaves well with respect to products. Applying the diagonal map we obtain a spectral sequence of bigraded rings. If $E \rightarrow B$ is an $H$-map there is an induced multiplication on $F$ and, when $R$ is a field, we have a spectral sequence of bigraded Hopf algebras.

Any map $f: B U \rightarrow B U$ may be regarded as a simple fibration $[24, \S 2.8]$ with $H^{*}(B U, R)$ of finite type over $R$ if $R$ is $p$-local. For such fibrations we may apply the beautiful Eilenberg-Moore spectral sequence collapse theorems of May ([14], [10]), Munkholm [18], and others ([3], [12], [22], [27]). The following special case of May's result [10, Theorem B] will suffice. We use the notation $\bigoplus M$ to denote the graded module associated to a bigraded module $M$.

4.1 TheOREM. If $f: B U \rightarrow B U$ is a fibration with fiber $F$, then there is a natural isomorphism of bigraded algebras

$$
E_{0} H^{*}(F, R) \cong \operatorname{Tor}_{H^{*}(B U, R)}\left(R, H^{*}(B U, R)\right)
$$

for some natural, nonpositive, decreasing filtration of $H^{*}(F, R)$. If $f$ is an $H$-map and $R$ is a field then this is an isomorphism of bigraded Hopf algebras. In either case, the map in filtration zero is induced by the canonical map

$$
H^{*}(B U, R) \otimes_{H^{*}(B U, R)} R \rightarrow H^{*}(F, R)
$$

induced by inclusion of the fiber. Finally, there is no additive extension problem and we obtain an isomorphism of graded $R$ modules

$$
H^{*}(F, R) \cong \bigoplus \operatorname{Tor}_{H^{*}(B U, R)}\left(R, H^{*}(B U, R)\right) .
$$

4.2. To determine the torsion products above we first compute $\operatorname{Tor}_{A_{n}^{*}}\left(R, A_{n}^{*}\right)$ for each $n$ prime to $p$ by means of the classical Koszul resolution. Let $\sigma: H^{i}(B U, R) \rightarrow H^{i-1}(U, R)$ denote the cohomology suspension; this is the composite

$$
H^{i}(B U, R) \stackrel{\pi^{*}}{\rightarrow} H^{i}(P B U, U ; R) \stackrel{\delta^{-1}}{\rightarrow} H^{i-1}(U, R)
$$

where $U \rightarrow P B U \rightarrow{ }^{\pi} B U$ is the path fibration. Then $\sigma\left(A_{n}^{*}\right) \subseteq H^{*}(U, R)$ is an exterior Hopf subalgebra $E\left\{\sigma a_{n, j}^{*} \mid j \geqslant 0\right\}$ on primitive generators $\sigma a_{n, j}^{*} \in$ $H^{2 n p^{j}-1}(U, R)$. The module $\sigma\left(A_{n}^{*}\right) \otimes_{R} A_{n}^{*}$ with differential generated by $\sigma a_{n, j}^{*} \rightarrow a_{n, j}^{*}$ is an $A_{n}^{*}$-free resolution of $R$ (compare $[3, \S 2]$ ). Moreover, the Hopf algebra structures on $A_{n}^{*}$ and $\sigma\left(A_{n}^{*}\right)$ induce one on the resolutions. Applying $\otimes_{f^{*}} A_{n}^{*}$ and checking the definition of product and coproduct for 
Tor, it follows that $\bigoplus$ Tor $_{A_{n}^{*}}\left(R, A_{n}^{*}\right)=H\left(\sigma A_{n}^{*} \otimes A_{n}^{*}, d\right)$ as a graded ring (and as a Hopf algebra if $f$ is an $H$-map and $R$ is a field) with differentials given by $\sigma a_{n, j}^{*} \rightarrow f^{*}\left(a_{n, j}^{*}\right)$. When $R$ is a field the external product on Tor is also an isomorphism [18] so we obtain a natural isomorphism

$$
\bigoplus \operatorname{Tor}_{H^{*}(B U, R)}\left(R, H^{*}(B U, R)\right) \cong \bigotimes_{n \text { prime to } p} \operatorname{Tor}_{A_{n}^{*}}\left(R, A_{n}^{*}\right)
$$

4.3 THEOREM. Let $f: B U \rightarrow B U$ be an $H$-map, $R=\mathrm{Z} / p$, and regard $A_{n}^{*}$ as a module over itself via $f^{*}$. Then

$$
\bigoplus \operatorname{Tor}_{A_{n}^{*}}\left(\mathrm{Z} / p, A_{n}^{*}\right) \cong E\left\{\sigma a_{n, j}^{*} \mid 0 \leqslant j<\delta_{n}\right\} \otimes_{\mathrm{Z} / p}\left(A_{p}^{*} / / \xi^{\delta_{n}} A_{n}^{*}\right)
$$

as Hopf algebras where $\xi: x \rightarrow x^{p}$ is the Frobenius map.

Proof. By $[23,1.5]$ there is an isomorphism of Hopf algebras

$$
\bigoplus \operatorname{Tor}_{A_{n}^{*}}\left(\mathrm{Z} / p, A_{n}^{*}\right) \cong \operatorname{Tor}_{\text {subker } f^{*}}(\mathrm{Z} / p, \mathrm{Z} / p) \otimes\left(A_{n}^{*} / / f^{*} A_{n}^{*}\right)
$$

where subker $f^{*}$ is the unique Hopf subalgebra of $A_{n}^{*}$ generating ker $f^{*}$ : $A_{n}^{*} \rightarrow A_{n}^{*}$. But by 3.7 , subker $f^{*}=\mathbf{Z} / p\left[a_{n, 0}^{*}, \ldots, a_{n, \delta_{n}-1}^{*}\right]$ and $f^{*} A_{n}^{*}=\xi^{\delta_{n}} A_{n}^{*}$, and the result follows by inspection of the Koszul resolution.

Thus by the Kunneth theorem there is an isomorphism of Hopf algebras

$$
\bigoplus E_{0} H^{*}(F, \mathbf{Z} / p) \cong \bigotimes_{n \text { prime to } p} E\left\{\sigma a_{n j}^{*} \mid 0 \leqslant j<\delta_{n}\right\} \otimes\left(A_{n}^{*} / / \xi^{\delta_{n}} A_{n}^{*}\right)
$$

Comparing this with the Eilenberg-Moore spectral sequence for the universal principal fibration (see e.g. [22]) and applying some standard Hopf algebra arguments, we may replace the term on the left with $H^{*}(F, \mathrm{Z} / p)$. This proves the following.

4.4 THEOREM. Let $f: B U \rightarrow B U$ be an $H$-map with fiber $F$. Then $H^{*}(F, Z / p)$ $\cong \bigotimes_{\text {nprime to } p} E\left\{\sigma a_{n, j}^{*} \mid 0 \leqslant j<\delta_{n}\right\} \otimes\left(A_{n}^{*} / / \xi^{\delta_{n}} A_{n}^{*}\right)$ as Hopf algebras. The in-. duced homomorphism of the inclusion $i: F \rightarrow B$ is given by the natural projections $A_{n}^{*} \rightarrow A_{n}^{*} / / \xi^{\delta_{n}} A_{n}^{*}$, while $\sigma a_{n, j}^{*}$ pulls back via the canonical map $U \rightarrow F$ to the cohomology suspension of $a_{n, j}^{*}$.

By 1.3 the fiber $F$ of an $H$-map $f: B U \rightarrow B U$ decomposes as an $H$ space into a product $F(0) \times F(2) \times \cdots \times F(2 p-4)$ where (in the notation of 1.4) $F(2 k)$ may be defined as the fiber of $f_{2 k}: B U \rightarrow B U$ or of $\tilde{f}_{2 k}: \Omega^{2 k} W \rightarrow \Omega^{2 k} W$. Applying 4.4 to $f_{2 k}$ we obtain the following.

4.5 Corollary. If $F=F(0) \times F(2) \times \cdots \times F(2 p-4)$ is the natural $H$ space decomposition of $F$, then

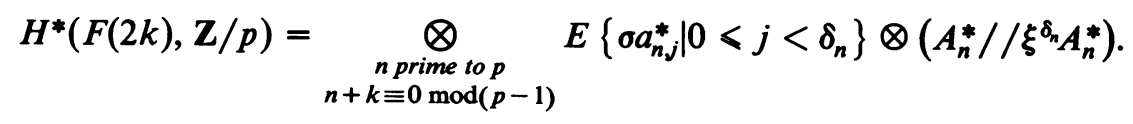


5. The Bockstein spectral sequence of the fiber. For any chain complex $(C, \partial)$ of abelian groups the long exact sequence in homology associated to the coefficient sequence $0 \rightarrow \mathbf{Z} \rightarrow^{p} \mathbf{Z} \rightarrow \mathbf{Z} / p \rightarrow 0$ may be regarded as an exact couple whose underlying graded spectral sequence is the Bockstein spectral sequence. The $E_{1}$ term is $E_{1}(C)=H_{*}(C, \mathrm{Z} / p)$ and the differential for $E_{r}$ is the $r$ th order Bockstein $\beta_{r}$ which may be described as follows. Given $\{c\} \in$ $H_{*}(C, Z / p)$ where $c \in C$ is a chain satisfying $\partial(c)=p^{r} c^{\prime}$, define $\beta_{r}\{c\}=$ $\left\{c^{\prime}\right\} \in H_{*}(C, \mathbf{Z} / p)$. This spectral sequence is discussed in considerable detail in [6] and [7]. We recall some useful facts.

5.1. If $x$ generates a direct summand $\mathrm{Z} / p^{r}$ in $H_{*}(C)$, then $j_{(r)}(y) \neq 0$ in $E_{r}(C)$ where $j_{(r)}: H_{*}(C) \rightarrow E_{r}(C)$ is the map induced by $j: H_{*}(C) \rightarrow$ $H_{*}(C, \mathbf{Z} / p)$.

5.2. $\operatorname{Kernel}\left(j_{r}\right)=p H_{*}(C)+T_{r-1}$ where $T_{r-1}$ is the subgroup of $H(C)$ annihilated by $p^{r-1}$.

5.3. $E_{\infty}(C)=\left(H_{*}(C) /\right.$ Torsion $) \otimes \mathrm{Z} / p$.

5.4. Given complexes $C$ and $D$, the canonical map $H_{*}(C, \mathrm{Z} / p) \otimes$ $H_{*}(D, \mathrm{Z} / p) \rightarrow H_{*}(C \otimes D, \mathrm{Z} / p)$ induces an isomorphism of chain complexes $E_{r}(C) \otimes E_{r}(D) \rightarrow E_{r}(C \otimes D)$ for all $r \geqslant 1$.

Now suppose $f: B U \rightarrow B U$ is an $H$-map with fiber $F$. Using 4.1 and 4.2 with $R=\mathbf{Z}_{(p)}$ it follows that $H^{*}\left(F, \mathbf{Z}_{(p)}\right)$ is the homology of the chain complex $\otimes_{n \text { prime to } p} \sigma\left(A_{n}^{*}\right) \otimes A_{n}^{*}$ with degree one differential defined by $\partial\left(\sigma\left(a_{n, j}^{*}\right)\right)=f^{*}\left(a_{n, j}^{*}\right)$. But since

$$
E_{r}\left(\bigotimes_{n \text { prime to } p}\left(\sigma\left(A_{n}^{*}\right) \otimes A_{n}^{*}\right)\right)=\bigotimes_{n \text { prime to } p} E_{r}\left(\sigma\left(A_{n}^{*}\right) \otimes A_{n}^{*}\right)
$$

by 5.4 , to describe the Bockstein spectral sequence of the space $F$ it will suffice to describe that of the component complexes $\sigma\left(A_{n}^{*}\right) \otimes A_{n}^{*}$. Of particular interest for geometric applications is a two-step complex of surplus $s$. This is a chain complex $\sigma\left(A_{n}^{*}\right) \otimes A_{n}^{*}$ in which the $H$-map $f$ inducing the differential as in 4.2 satisfies $s(f)_{n p^{k}}=s>0$ for all $k<\delta_{n}$.

5.5 TheOREM. Let $\sigma\left(A_{n}^{*}\right) \otimes A_{n}^{*}$ be a two-step complex of surplus $s$. For $r \leqslant s$ the Bockstein spectral sequence is given by

$$
E_{r} \cong E\left\{\sigma a_{n, 0}^{*}, \ldots, \sigma a_{n, \delta_{n}-1}^{*}\right\} \otimes A_{n}^{*} / / \xi^{\delta_{n}} A_{n}^{*} \otimes \mathrm{Z} / p
$$

If $r \geqslant s$ we have

$$
E_{r} \cong E\left\{\sigma a_{n, r-s}^{*}, \ldots, \sigma a_{n, r-s+\delta_{n}-1}^{*}\right\} \otimes A_{n}^{*}(r-s) / / \xi^{\delta_{n}} A_{n}^{*}(r-s) \otimes \mathbf{Z} / p
$$

where $A_{n}^{*}(k)=\mathbf{Z}_{(p)}\left[a_{n, k}^{*}, a_{n, k+1}^{*}, \ldots\right]$.

Proof. For convenience we may first suppose that the characteristic subsequence $\lambda_{n}, \lambda_{p n}, \ldots$ used to define the differential on $\sigma\left(A_{n}^{*}\right) \otimes A_{n}^{*}$ is given by $p^{s}, p^{s+1}, \ldots, p^{s+\delta_{n}-1}, p^{\delta_{n}}, p^{\delta_{n}} \ldots$ By 3.6 we may choose elements 
$c_{k}=\sigma a_{n, k}^{*}-\sum_{i<k} \sigma\left(a_{n, i}^{*}\right) \otimes x_{i}$ for suitable $x_{i} \in A_{n}^{*}$ such that $\partial\left(c_{k}\right)=$ $p^{s} T_{k}\left(a_{n, 0}^{*}, \ldots, a_{n, k}^{*}\right)$ if $k<\delta_{n}$ and $\partial\left(c_{k}\right)=\left(a_{n, k-\delta_{n}}^{*}\right)^{p_{n}^{*}}-\xi_{k-\delta_{n}}\left(a_{n, 1}^{*}, \ldots, a_{n, k}^{*}\right)$ for $k \geqslant \delta_{n}$. Then setting $b_{0}=c_{0}, b_{k}=c_{k}-b_{0}\left(a_{n, 0}^{*}\right)^{p^{k-1}}$ $-\cdots-b_{k-1}\left(a_{n, k-1}^{*}\right)^{p-1}$ for $0<k<\delta_{n}$, and $b_{k}=c_{k}$ for $k \geqslant \delta_{n}$, it follows that we may rewrite $\sigma\left(A_{n}^{*}\right) \otimes A_{n}^{*}$ as a complex $C(0)=E\left\{b_{0}, b_{1}, \ldots\right\} \otimes A_{n}^{*}$ with differential $\partial_{0, s}$ defined by $\partial_{0, s}\left(b_{k}\right)=p^{k+s} a_{n, k}^{*}$ for $k<\delta_{n}$ and $\partial_{0, s}\left(b_{k}\right)=$ $\left(a_{n, k-\delta_{n}}\right)^{p_{n}}-\xi_{k-\delta_{n}}\left(a_{n, 1}^{*}, \ldots, a_{n, k}^{*}\right)$ if $k \geqslant \delta_{n}$.

For each $k \geqslant 0$ let $C(k)$ denote the free $\mathbf{Z}_{(p)}$ module $E\left\{b_{k}, b_{k+1}, \ldots\right\} \otimes$ $A_{n}^{*}(k)$ where $b_{j}$ is, as above, an exterior generator of degree $n p^{j}-1$. On $C(0)$ we have already defined differentials $\partial_{0, s}$ for each $s>0$. We may similarly define $\partial_{k, s}$ on $C(k)$ by

$$
\begin{gathered}
\partial_{k, s}\left(b_{j}\right)=p^{j-k+s} a_{n, j}^{*} \quad \text { if } k \leqslant j<k+\delta_{n}, \\
\partial_{k, s}\left(b_{j}\right)=\left(a_{n, j-\delta_{n}}^{*}\right)^{\delta_{n}}-\xi_{j-k-\delta_{n}}\left(a_{n, k+1}^{*}, \ldots, a_{n, j}^{*}\right) \quad \text { if } j \geqslant k+\delta_{n} .
\end{gathered}
$$

Assertion 1. $E_{r}\left(C(0), p^{i} \partial_{0, s-i}\right) \cong E_{r}\left(C(0), p^{i-1} \partial_{0, s-i+1}\right)$ for all $i=1, \ldots, s$ -1 and $r>i$.

Assertion 2. $E_{r}\left(C(k), p^{k} \partial_{k, 1}\right) \cong E_{r}\left(C(k+1), p^{k+1} \partial_{k+1,1}\right)$ if $r>k+1$.

Assuming these for the moment the proof proceeds as follows. Clearly $E_{i}\left(C(k), p^{i} \partial_{k, s}\right)=C(k)$ with $i$ th Bockstein the $\bmod p$ reduction of $\partial_{k, s}$. Thus

$$
E_{i+1}\left(C(k), p^{i} \partial_{k, s}\right)=E\left\{b_{k}, \ldots, b_{k+\delta_{n}-1}\right\} \otimes A_{n}^{*}(k) / / \xi^{\delta_{n}} A_{n}^{*}(k) \otimes \mathrm{Z} / p
$$

by the argument of 4.3. From the definition of the elements $b_{j}$ this is isomorphic to $E\left(\sigma a_{n, k}^{*}, \ldots, \sigma a_{n, k+\delta_{n}-1}^{*}\right) \otimes A_{n}^{*}(k) / / \xi^{\delta_{n}} A_{n}^{*}(k) \otimes \mathbf{Z} / p$. Thus if $r \leqslant s$ it follows by Assertion 1 that

$$
\begin{aligned}
E_{r}\left(C(0), \partial_{0, s}\right) & \cong E_{r}\left(C(0), p^{r-1} \partial_{0, s-r+1}\right) \\
& \cong E\left\{\sigma a_{n, 0}^{*}, \ldots, \sigma a_{n, \delta_{n}-1}^{*}\right\} \otimes A_{n}^{*} / / \xi^{\delta_{n}} A_{n}^{*} \otimes \mathbf{Z} / p .
\end{aligned}
$$

When $r \geqslant s$ it follows by Assertions 1 and 2 that

$$
\begin{aligned}
E_{r}\left(C(0), \partial_{0, s}\right) \cong E_{r}\left(C(0), p^{s-1} \partial_{0,1}\right) \\
\quad \cong E_{r-s+1}\left(C(0), \partial_{0,1}\right) \\
\quad \cong E_{r-s+1}\left(C(r-s), p^{r-s} \partial_{r-s, 1}\right) \\
\quad \cong E\left\{\sigma a_{n, r-s}, \ldots, \sigma a_{n, r-s+\delta_{n}-1}\right\} \otimes A_{n}^{*}(r-s) / / \xi^{\delta_{n}} A_{n}^{*}(r-s) \otimes \mathbf{Z} / p .
\end{aligned}
$$

To prove Assertion 1 it suffices to check that $E_{r}\left(C(0), p \partial_{0, s-i}\right) \cong$ $E_{r}\left(C(0), \partial_{0, s-i+1}\right)$ for $r>1$ and $i=1, \ldots, s-1$. Define a chain map $\varphi$ : $\left(C(0), \partial_{0, s-i+1}\right) \rightarrow\left(C(0), p \partial_{0, s-i}\right)$ by setting $\varphi=1$ on $A_{n}^{*}, \varphi\left(b_{i}\right)=b_{i}$ if $i<\delta_{n}$, and $\varphi\left(b_{i}\right)=p b_{i}$ if $i \geqslant \delta_{n}$. Then $\varphi$ induces an isomorphism on $E_{2}$. For as noted 
above,

$$
\begin{aligned}
E_{2}\left(C(0), p \partial_{0, s-i}\right) & =E_{1}\left(C(0), \partial_{0, s-i}\right) \\
& =E\left\{b_{0}, \ldots, b_{\delta_{n}-1}\right\} \otimes A_{n}^{*} / / \xi^{\delta_{n}} A_{n}^{*} \otimes \mathbf{Z} / p .
\end{aligned}
$$

But by the same argument we have $E_{1}\left(C(0), \partial_{0, s-i+1}\right)=E\left\{b_{0}, \ldots, b_{\delta_{n}-1}\right\} \otimes$ $A_{n}^{*} / / \xi^{\delta_{n}} A_{n}^{*} \otimes \mathrm{Z} / p$ with the entire homology group lying in the kernel of the first Bockstein.

The second assertion is proved similarly. We will define a chain map $\varphi$ : $\left(C(k+1), p \partial_{k+1,1}\right) \rightarrow\left(C(k), \partial_{k, 1}\right)$ which induces an isomorphism on $E_{2}$. Let $\left.\varphi\right|_{A(k+1)}$ be the canonical inclusion $A(k+1) \subseteq A(k)$, define $\varphi\left(b_{j}\right)=b_{j}$ for $k+1<j<k+\delta_{n}$, and

$$
\varphi\left(b_{k+\delta_{n}}\right)=p b_{k+\delta_{n}}-b_{k} \otimes\left(a_{n, k}^{*}\right)^{p^{\delta_{n}-1}}-\cdots-b_{k+\delta_{n}-1} \otimes\left(a_{n, k+\delta_{n}-1}^{*}\right)^{p-1} \text {. }
$$

Next abbreviate $\xi_{j}\left(a_{n, k+1}^{*}, \ldots, a_{n, k+j+\delta_{n}}^{*}\right)=\xi$ and $\xi_{j-1}\left(a_{n, k+2}^{*}, \ldots, a_{n, k+j+\delta_{n}}^{*}\right)$ $=\hat{\xi}_{j-1}$, and note that the defining relation 3.4 yields

$$
p T_{k-\delta_{n}-1}\left(\hat{\xi}_{0}, \ldots, \hat{\xi}_{k-\delta_{n}-1}\right)=T_{k-\delta_{n}}\left(\xi_{0}, \ldots, \xi_{k-\delta_{n}}\right) \text {. }
$$

Then using the $p$-divisibility of $\xi_{0}$ and the binomial theorem (compare the proof of 2.4) it follows inductively that $\hat{\xi}_{j-1}=\xi_{j}+\eta_{j} \xi_{0}$ for some $\eta_{j} \in A_{n}^{*}(k)$. We complete the definition of $\varphi$ by setting $\varphi\left(b_{k+\delta_{n}+j}\right)=p b_{k+\delta_{n}+j}+\eta_{j} x$ for all $j>0$ where $x=p b_{k+\delta_{n}}-b_{k}\left(a_{n, k}^{*}\right)^{p^{\delta_{n-1}}}$ satisfies $\partial_{k, 1}(x)=p \xi_{0}$.

To compute $\varphi_{*}$ on $E_{2}$ recall first that

$$
\begin{aligned}
& E_{2}\left(C(k+1), p \partial_{k+1,1}\right) \\
& \quad=E\left\{b_{k+1}, \ldots, b_{k+\delta_{n}}\right\} \otimes A_{n}^{*}(k+1) / / \xi^{\delta_{n}} A_{n}^{*}(k+1) \otimes \mathbf{Z} / p
\end{aligned}
$$

and

$$
E_{1}\left(C(k), \partial_{k, 1}\right)=E\left\{b_{k}, \ldots, b_{k+\delta_{n}-1}\right\} \otimes A_{n}^{*}(k) / / \xi^{\delta_{n}} A_{n}^{*}(k) \otimes \mathbf{Z} / p
$$

as before. The first Bockstein on the latter ring is defined by the conditions $\beta_{1}\left(b_{k}\right)=a_{k}$ and $\beta_{1}\left(b_{k+1}\right)=\cdots=\beta_{1}\left(b_{k+\delta_{n}-1}\right)=\beta_{1}\left(A_{n}^{*}(k) / / \xi^{\delta_{n}} A_{n}^{*}(k)\right)=0$. It follows that $\operatorname{Ker} \beta_{1}$ is the subring generated by $b_{k+1}, \ldots, b_{k+\delta_{n}-1}, b_{k} \otimes$ $\left(a_{n, k}^{*}\right)^{p_{n-1}}$, and $A_{n}^{*}(k)$, while $\operatorname{Im}\left(\beta_{1}\right)=a_{n, k}^{*} \cdot\left(\operatorname{Ker} \beta_{1}\right)$. By a change of basis we may thus write $\operatorname{Ker} \beta_{1}=E\left\{b_{k+1}^{\prime}, \ldots, b_{k+\delta_{n}}^{\prime}\right\} \otimes A_{n}^{*}(k) / / \xi^{\delta_{n}} A_{n}^{*}(k) \otimes \mathbf{Z} / p$ where $b_{j}^{\prime}=b_{j}$ if $j<k+\delta_{n}$ and $b_{k+\delta_{n}}^{\prime}=p b_{k+\delta_{n}}-b_{k} \otimes\left(a_{n, k}^{*}\right)^{p^{\delta_{n}-1}}$ -.. - $b_{k+\delta_{n}-1} \otimes\left(a_{n, k+\delta_{n}-1}^{*}\right)^{p-1}$. Thus $E_{2}\left(C(k), \partial_{k, 1}\right)=$ $E\left\{b_{k+1}^{\prime}, \ldots, b_{k+\delta_{n}}^{\prime}\right\} \otimes\left(A_{n}^{*}(k+1) / / \xi^{\delta_{n}} A_{n}^{*}(k+1)\right)$ with $\varphi$ inducing an isomorphism on $E_{2}$.

The Bockstein spectral sequence for the complex $C=\sigma\left(A_{n}^{*}\right) \otimes A_{n}^{*}$ with arbitrary surplus $s_{n}, s_{n p}, \ldots, s_{n p^{s_{n}-1}}$ is, unfortunately, still a mess. If $s=$ $\min \left\{s_{n}, \ldots, s_{n p_{n}-1}\right\}$ and $C^{1}=\sigma\left(A_{n}^{*}\right) \otimes A_{n}^{*}$ is the complex of constant surplus $s$ obtained by dividing the characteristic sequence for $C$ by appropriate 
powers of $p$, then applying 5.1, 5.2, and 5.3 to the computation 5.5 yields reasonably explicit information about the torsion group $H\left(C^{1}\right)$. In particular cases one can then profitably compare $H(C)$ and $H\left(C^{1}\right)$ by studying, via the mapping cone construction, the homology of the chain map $\varphi: C \rightarrow C^{1}$ defined by

$$
\begin{gathered}
\varphi\left(\sigma a_{n, j}^{*}\right)=p^{s_{n j}-s} \sigma a_{n, j}^{*} \text { for } j<\delta_{n}, \\
\varphi\left(\sigma a_{n, j}^{*}\right)=\sigma a_{n, j}^{*} \text { for } j \geqslant \delta_{n},
\end{gathered}
$$

and $\left.\varphi\right|_{A_{n}^{*}}=1$. Such considerations are omitted here in the hope the general case will soon be brought under control.

6. An important special case. The $\mathbf{Z}_{(p)}$ cohomology of the fiber of an $H$-map $f: B U \rightarrow B U$ with index never exceeding 1 admits a direct description. Such an explicit computation, though less transparent than the Bockstein spectral sequence computation of the previous section, will be necessary in a subsequent study of the classifying space of smoothing theory.

We first suppose that $f: B U \rightarrow B U$ is an $H$-map with characteristic sequence $\lambda$ such that, for a fixed $n$ prime to $p, \delta_{n}(f)=1$ and $\lambda_{n}=p^{m} w$ for some unit $w \in \mathbf{Z}_{(p)}$. Regarding $A_{n}^{*}$ as a module over itself via $f^{*}$ as usual, we describe $\bigoplus$ Tor $_{A_{n}^{*}}\left(\mathbf{Z}_{(p)}, A_{n}^{*}\right)$ as both a $\mathbf{Z}_{(p)}$ module and an algebra.

6.1 THEOREM. There is an isomorphism of graded algebras

$$
\bigoplus \operatorname{Tor}_{A_{n}^{*}}\left(\mathbf{Z}_{(p)}, A_{n}^{*}\right) \cong A_{n}^{*} / / f^{*} A_{n}^{*}=A_{n}^{*} / I
$$

where $I$ is the $A_{n}^{*}$ ideal generated by

$$
\lambda_{n} a_{n, 0}^{*},\left(a_{n, 0}^{*}\right)^{p}-p w_{1} a_{n, 1}^{*},\left(a_{n, 1}^{*}\right)^{p}-p w_{2} a_{n, 2}^{*}, \ldots
$$

for certain units $w_{1}, w_{2}, \ldots \in \mathbf{Z}_{(p)}$. The submodule of elements of degree $2 r$ is isomorphic to $\bigoplus_{\alpha} \mathrm{Z} /$ ind $(\alpha)$ where $\alpha=\left(\alpha_{1}, \alpha_{2}, \ldots\right)$ ranges over all sequences of nonnegative integers of weight $r$ each of whose nonzero entries is $<(p-1)$ and lies in the subsequence $\alpha_{n}, \alpha_{n p}, \alpha_{n p^{2}}, \ldots$, and where $\operatorname{ind}(\alpha)=p^{m+j}$ if $\alpha_{n p^{j}}$ is the first nonzero entry of $\alpha$.

Proof. By the definition of torsion product (compare e.g. [3]) we have that $\operatorname{Tor}_{A^{*}}^{0 *}\left(\mathbf{Z}_{(p)}, A_{n}^{*}\right)=\mathbf{Z}_{(p)} \otimes_{f^{*}} A_{n}^{*}=A_{n}^{*} / / f^{*} A_{n}^{*}$ where the quotient module $A_{n}^{*} / h^{*} A_{n}^{*}$ is torsion because $f^{*}$ is a rational isomorphism. But since $\left(A_{n}^{*} / / f^{*} A_{n}^{*}\right) \otimes \mathbf{Z} / p \cong A_{n}^{*} \otimes \mathbf{Z} / p / / f^{*} A_{n}^{*} \otimes \mathbf{Z} / p$ is a vector space with one generator in each dimension $n j$ by 3.7 , it follows by 4.4 and inspection that

$$
\begin{aligned}
& \operatorname{Tor}_{A_{n}^{*} \otimes \mathbf{Z} / p}\left(\mathrm{Z} / p, A_{n}^{*} \otimes \mathbf{Z} / p\right) \cong E\left\{\sigma a_{n, 0}^{*}\right\} \otimes A_{n}^{*} / / \xi A_{n}^{*} \otimes \mathbf{Z} / p \\
& \cong\left(A_{n}^{*} / / f^{*} A_{n}^{*} \otimes \mathbf{Z} / p\right) \oplus \operatorname{Tor}_{1}\left(\left(A_{n}^{*} / / f^{*} A_{n}^{*}\right), \mathbf{Z} / p\right) .
\end{aligned}
$$

But by universal coefficients $\operatorname{Tor}_{A_{n}^{*} \otimes \mathbf{Z} / p}\left(\mathbf{Z} / p, A_{n}^{*} \otimes \mathbf{Z} / p\right)$ is isomorphic to 
$\left(\operatorname{Tor}_{A_{n}^{*}}\left(\mathbf{Z}_{(p)}, A_{n}^{*}\right) \otimes \mathbf{Z} / p\right) \oplus \operatorname{Tor}_{1}\left(\operatorname{Tor}_{A_{n}^{*}}\left(\mathbf{Z}_{(p)}, A_{n}^{*}\right), \mathbf{Z} / p\right)$. Thus $\operatorname{Tor}_{A_{n}^{*}}^{s, t}\left(\mathbf{Z}_{(p)}, A_{n}^{*}\right)$ $=0$ if $s \neq 0$ and hence

$$
\operatorname{Tor}_{A_{n}^{*}}\left(\mathbf{Z}_{(p)}, A_{n}^{*}\right)=\bigoplus \operatorname{Tor}_{A_{n}^{*}}\left(\mathbf{Z}_{(p)}, A_{n}^{*}\right)=A_{n}^{*} / / f^{*} A_{n}^{*} \text {. }
$$

For each $j \geqslant 0$ let $I_{j}$ denote the $A_{n}^{*}$ ideal generated by $f^{*}\left(a_{n, 0}^{*}\right), \ldots, f^{*}\left(a_{n, j}^{*}\right)$ and assume inductively that $I_{j-1}$ is generated by $\lambda_{n} a_{n, 0}^{*},\left(a_{n, 0}^{*}\right)^{p}-$ $w_{1} p a_{n, 1}^{*}, \ldots,\left(a_{n, j-2}^{*}\right)^{p}-w_{j-1} p a_{n, j-1}^{*}$ for some units $w_{1}, \ldots, w_{j-1} \in \mathbf{Z}_{(p)}$. It follows from 3.4 and 3.5 that $f^{*}\left(a_{n, j}^{*}\right)=v_{j}\left(\left(a_{n, j-1}^{*}\right)^{p}-\xi_{j-1}\left(a_{n, 1}^{*}, \ldots, a_{n, j}^{*}\right)\right)+x$ where $x \in I_{j-1}$ and $v_{j}=p / \lambda_{n p}$, a unit in $\mathbf{Z}_{(p)}$. By definition $\xi_{j-1}$ is a $p$-divisible polynomial given by $\xi_{j-1}\left(a_{n, 1}^{*}, \ldots, a_{n, j}^{*}\right)=-p a_{n, j}^{*}-$ $p^{1-j} T_{j-2}\left(\xi_{0}^{p}, \ldots, \xi_{j-2}^{p}\right)$. If $j=1$ the last term on the right vanishes, and if $j=2$ this becomes $\xi_{1}\left(a_{n, 1}^{*}, a_{n, 2}^{*}\right)=-p a_{n, 2}^{*}+p^{p-1}\left(a_{n, 1}^{*}\right)^{p}$. In general, since $\xi_{j-1}$ is homogeneous of degree $n p^{j}$ and since the degrees of $a_{n, 2}^{*}, \ldots, a_{n, j}^{*}$ are multiples of $n p^{2}$, we may regard $\xi_{j-1}$ as a polynomial in $\left(a_{n, 1}^{*}\right)^{p}, a_{n, 2}^{*}, \ldots, a_{n, j}^{*}$. Thus $\xi_{j-1} \equiv-p a_{n, j}^{*}+p \xi^{\prime}\left(a_{n, 2}^{*}, \ldots, a_{n, j-1}^{*}\right) \bmod I_{j-1}$ for some polynomial $\xi^{\prime}$. As before, regard $\xi^{\prime}$ as a polynomial in $\left(a_{n, 2}^{*}\right)^{p}, a_{n, 3}^{*}, \ldots, a_{n, j}^{*}$ and continue inductively. Thus we may write

$$
\xi_{j-1}\left(a_{n, 1}^{*}, \ldots, a_{n, j}^{*}\right) \equiv-p a_{n, j}^{*}+p \eta\left(a_{n, j-1}^{*}\right)^{p} \bmod I_{j-1}
$$

for some $\eta \in \mathbf{Z}_{(p)}$. It follows that $I_{j}$ is generated over $A_{n}^{*}$ by $I_{j-1}$ and $\left(a_{n, j-1}^{*}\right)^{p}-w_{k} p a_{n, j}^{*}$ where $w_{k}=1 /(\eta p-1)$ and the first part of 6.1 is established.

It follows from the work above that any monomial in $A_{n}^{*}$ is congruent modulo $I=I_{1} \cup I_{2} \cup \ldots$ to some multiple of a monomial in "reduced form"

$$
\left(\left(a_{n}^{*}\right)^{\left(k_{00} \ldots, k_{j}\right)}=\left(a_{n, 0}^{*}\right)^{k_{0}} \ldots\left(a_{n, j}^{*}\right)^{k_{j}} \text { where } k_{i}<p \text { for all } i\right) .
$$

Moreover this monomial is unique since distinct reduced monomials have different degrees. We must show that $\left(a_{n}^{*}\right)^{\left(0, \ldots, 0, k_{i}, \ldots, k_{j}\right)}$ has order precisely $p^{m+i}$ if $k_{i} \neq 0$. From the congruences

$$
\begin{aligned}
p^{i}\left(a_{n}^{*}\right)^{\left(0, \ldots, 0, k_{i}, \ldots, k_{j}\right)} & \equiv\left(p^{i-1} / w_{i}\right)\left(a_{n}^{*}\right)^{\left(0, \ldots, 0, p, k_{i}-1, \ldots, k_{j}\right)} \equiv \ldots \\
& \equiv\left(1 / w_{1} \cdots w_{i}\right)\left(a_{n}^{*}\right)^{\left(p, p-1, \ldots, p-1, k_{i}-1, k_{i+1}, \ldots, k_{j}\right)}
\end{aligned}
$$

$\bmod I$ it will suffice to show that any monomial $\left(a_{n}^{*}\right)^{\left(k_{0}, \ldots, k_{j}\right)}$ with $0<k_{0} \leqslant p$ and $k_{i}<p$ for $i>0$ has order $p^{m}$ in $A_{n}^{*} / I$. Thus suppose

$$
\eta\left(a_{n}^{*}\right)^{\left(k_{0}, \ldots, k_{j}\right)}=\lambda_{n} a_{n, 0}^{*} x_{0}+\sum_{i=0}^{l}\left(\left(a_{n, i}^{*}\right)^{p}-w_{i} p a_{n, i}^{*}\right) x_{i}
$$

for some $x_{0}, \ldots, x_{l} \in A_{n}^{*}$ and $\eta \in \mathbf{Z}_{(p)}$, and consider the evaluation map $E$ : $A_{n}^{*} \rightarrow \mathbf{Z}_{(p)}$ sending

$$
a_{n, k}^{*} \rightarrow\left(p^{p^{k}-p^{k-1}-\cdots-p-1}\right) /\left(w_{1}^{p^{k-1}} w_{2}^{p^{k-2}} \cdots w_{k-1}^{p} w_{k}\right) .
$$


This map is defined so that $E\left(\left(a_{n, k-1}^{*}\right)^{p}-w_{k} p a_{n, k}^{*}\right)=0$ for all $k>0$. This implies that among all monomials $\left(a_{n}^{*}\right)^{\left(l_{0}, \ldots, l_{j}\right)}$ of a given degree the one whose $E$ image has the lowest $p$ divisibility is the monomial in reduced form. Applying $E$ to (*) above we obtain $\eta E\left(\left(a_{n}^{*}\right)^{\left(k_{0}, \ldots, k_{j}\right)}\right)=\lambda_{n} E\left(a_{n, 0}^{*} x_{0}\right)$. Thus if $k_{0}<p, \eta$ must be divisible by at least $p^{m}$. If $k_{0}=p$, then both $\left(a_{n}^{*}\right)^{\left(k_{0}, \ldots, k_{j}\right)}$ and $a_{n, 0}^{*} x_{0}$ have a factor $\left(a_{n, 0}^{*}\right)^{p}$, so again $p^{m}$ divides $\eta$.

Using universal coefficients we can now combine the calculation above for various values of $n$ to prove Theorem $D$ of the introduction.

Proof of Theorem D. For each finite subset $S^{\prime} \subseteq S$ let $C_{S^{\prime}}$ denote the complex $\otimes_{n \in S^{\prime}} \sigma A_{n}^{*} \otimes A_{n}^{*}$ with differential defined via $f^{*}$ as in 4.2 , and let $T_{S^{\prime}} \subseteq T$ consist of those sequences $\alpha$ whose nonzero entries lie in some subsequence $\alpha_{n}, \alpha_{p n}, \ldots$ for $n \in S^{\prime}$. We will show by induction on the size of $S^{\prime}$ that

$$
H_{m}\left(C_{S^{\prime}}\right) \cong \bigoplus_{j=1}^{\infty} \bigoplus_{i=0}^{j-1} \bigoplus_{\substack{\alpha \in T_{S^{\prime}} \\ l(\alpha)=j \\ 2 w(\alpha)=m+i}}(\mathrm{Z} / \text { ind } \alpha)^{\left(i^{-1}\right)} .
$$

This proves 6.2 since, for each $m>0, H^{m}\left(F, \mathbf{Z}_{(p)}\right)=H_{m}\left(C_{S^{\prime}}\right)$ for some finite $S^{\prime} \subseteq S$.

If $S^{\prime}$ consists of a single element, then $l(\alpha)=1$ for any $\alpha \in T_{S^{\prime}}$ and the formula 6.2 reduces to that of 6.1. Thus suppose $S^{\prime} \subseteq S$ is some finite set for which (6.2) holds and choose $n \in S \backslash S^{\prime}$. By the Künneth formula

$$
\begin{aligned}
H_{m}\left(C_{S^{\prime} \cup\{n\}}\right) & =H_{m}\left(C_{S^{\prime}} \otimes C_{\{n\}}\right) \\
& \cong \underbrace{\bigoplus_{m^{\prime}+m^{\prime}=m} H_{m^{\prime}}\left(C_{S^{\prime}}\right) \otimes H_{m^{\prime \prime}}\left(C_{\{n\}}\right)}
\end{aligned}
$$

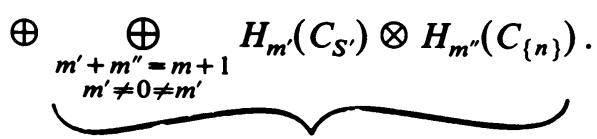

(b)

This uses the fact that the reduced groups $\tilde{H}_{*}\left(C_{S^{\prime}}\right)$ and $\tilde{H}_{*}\left(C_{\{n\}}\right)$ are torsion. But for any $\alpha \in T_{S^{\prime} \cup\{n\}}$ there are isomorphisms

$$
\begin{aligned}
\mathbf{Z} / \text { ind } \alpha & \cong \mathbf{Z} / \min \left(\text { ind } \alpha^{\prime}, \text { ind } \alpha^{\prime \prime}\right) \\
& \cong \mathbf{Z} / \text { ind } \alpha^{\prime} \otimes \mathbf{Z} / \text { ind } \alpha^{\prime \prime}
\end{aligned}
$$

where $\alpha=\alpha^{\prime}+\alpha^{\prime \prime}$ for some unique $\alpha^{\prime} \in T_{S}, \alpha^{\prime \prime} \in T_{\{n\}}$.

Then applying the inductive assumption, the subgroup of (a) above corresponding to $m^{\prime}=0$ or $m^{\prime \prime}=0$ is given by 


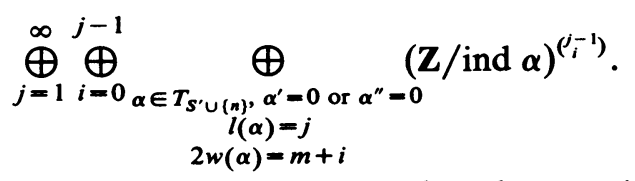

When $\alpha^{\prime \prime} \neq 0$ we have $l\left(\alpha^{\prime}\right)=l(\alpha)-1$ so that the remaining contribution from (a) is

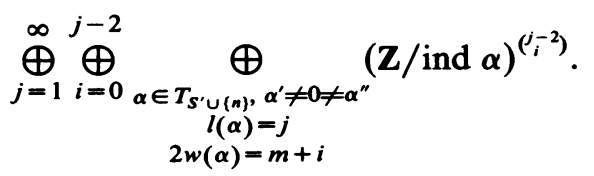

Similarly, applying the inductive assumption to (b) yields

$$
\begin{aligned}
& \bigoplus_{j=1}^{\infty} \bigoplus_{\substack{i=0 \\
\alpha \in T_{S^{\prime} \cup(n), \alpha^{\prime} \neq 0 \neq \alpha^{\prime \prime}}(\mathrm{Z} /(\alpha)=j \\
2 w(\alpha)=m+i+1}}(\mathrm{ind} \alpha)^{\left(j_{i}^{-2}\right)} \\
& =\bigoplus_{j=1}^{\infty} \bigoplus_{i=1}^{j-1} \bigoplus_{\substack{\alpha \in T_{s^{\prime}} \cup(n), \alpha^{\prime} \neq 0 \neq \alpha^{\prime \prime} \\
l(\alpha)=j \\
w(\alpha)=m+i}}(\mathrm{Z} / \text { ind } \alpha)^{\left(j_{i-1}^{j-2}\right)} .
\end{aligned}
$$

Then adding $\left(a_{1}\right),\left(a_{2}\right)$, and $\left(b_{1}\right)$ and substituting the identity

$$
\left(\begin{array}{c}
j-2 \\
i
\end{array}\right)+\left(\begin{array}{c}
j-2 \\
i-1
\end{array}\right)=\left(\begin{array}{c}
j-1 \\
i
\end{array}\right)
$$

we obtain the desired formula 6.2 for the set $S^{\prime} \cup\{n\}$.

We conclude with a remark about nontorsion elements. Most of the results in this paper involve an $H$-map $f: B U \rightarrow B U$ whose characteristic sequence has only nonzero entries. Equivalently, the reduced $\mathbf{Z}_{(p)}$ cohomology of the fiber of $f$ is torsion. If $\lambda$ has trivial entries, however, there is a reasonably simple algorithm for computing the rank of the cohomology. For any set $S$ of positive integers and for any positive integers $k$ and $n$, denote by $p_{S}(k, n)$ the number of distinct ways of writing $n$ as a sum of integers from the set $S$ (an element may be used more than once) in which exactly $k$ different elements from $S$ are used. For example, if $S$ is the set of all positive integers then $p_{S}(1, n)+p_{S}(2, n)+\ldots$ is the number of partitions of $n$. Let $p_{s}(k, n)=0$ if $n$ is not an integer.

6.4 THEOREM. Let $F: B U \rightarrow B U$ be an $H$-map with characteristic sequence $\lambda$ and fiber $F$, and write $S$ for the set of all indices $n$ such that $\lambda_{n}=0$. Then for every $m>0$ the $\mathbf{Z}_{(p)}$ rank of $H^{m}\left(F, \mathbf{Z}_{(p)}\right)$ is given by

$$
\sum_{i, j>0}\left(\begin{array}{c}
i+j \\
i
\end{array}\right) p_{s}(i+j,(i+m) / 2) \text {. }
$$

We omit the proof which is a straightforward counting argument involving the rational Eilenberg-Moore spectral sequence of $f$. 


\section{REFERENCES}

1. J. F. Adams, Lectures on generalized cohomology, Category Theory, Homology Theory, and their Applications. III, Lecture Notes in Math. No. 99, Springer-Verlag, Berlin and New York, 1969.

2. J. F. Adams and S. Priddy, Uniqueness of BSO, Proc. Cambridge Philos. Soc. 80 (1976), 475-509.

3. P. F. Baum, On the cohomology of homogeneous spaces, Topology 7 (1968), 15-38.

4. A. Borel and J. P. Serre, Groups de Lie et puissances réduite de Steenrod, Amer. J. Math. 75 (1953), 409-448.

5. R. Bott and J. Milnor, On the parallelizability of the spheres, Bull. Amer. Math. Soc. 64 (1958), 87-89.

6. W. Browder, Torsion in H-spaces, Ann. of Math. (2) 74 (1961), 24-51.

7. Higher torsion in H-spaces, Trans. Amer. Math. Soc. 108 (1963), 353-375.

8. H. Cartan and S. Eilenberg, Homological algebra, Princeton Univ. Press, Princeton, N. J., 1956.

9. S. Eilenberg and J. C. Moore, Homology and fibrations. I, Comment. Math. Helv. 40 (1966), 199-236.

10. V. K. A. M. Gugenheim and J. P. May, On the theory and application of differential torsion products, Mem. Amer. Math. Soc. No. 142, 1974.

11. D. Husemoller, The structure of the Hopf algebra $H_{*}(B U)$ over a $\mathbf{Z}_{(p)}$-algebra, Amer. J. Math. 43 (1971), 329-349.

12. D. Husemoller, J. C. Moore and J. Stasheff, Differential homological algebra and homogeneous spaces, J. Pure Appl. Algebra 5 (1974), 113-185.

13. A. Liulevicius, On characteristic classes, Nordic Summer School Notes, Aarhus Universitet, 1968.

14. J. P. May, The cohomology of principal bundles, homogeneous spaces, and two stage Postnikov systems, Bull. Amer. Math. Soc. 74 (1968), 334-339.

15. Verlag, Berlin and New York, 1977.

16. J. Milnor and J. Stasheff, Characteristic classes, Ann. of Math. Studies No. 76, Princeton Univ. Press, Princeton, N. J., 1974.

17. J. Milnor and J. C. Moore, On the structure of Hopf algebras, Ann. of Math. 81 (1965), 211-264.

18. H. Munkholm, The Eilenberg-Moore spectral sequence and strongly homotopy multiplicative maps, J. Pure Appl. Algebra 5 (1974), 1-50.

19. F. P. Peterson, The mod p homotopy type of BSO and F/PL, Bol. Soc. Mat. Mexicana 14 (1969), 22-27.

20. H. Rademacher, Lectures on elementary number theory, Blaisdell, Waltham, Mass., 1964.

21. D. Ravenel and W. S. Wilson, Bipolynomial Hopf algebras, J. Pure Appl. Algebra 4 (1974), $41-45$.

22. L. Smith, Homological algebra and the Eilenberg-Moore spectral sequence, Trans. Amer. Math. Soc. 129 (1967), 58-93.

23. , Cohomology of stable two stage Postnikov systems, Illinois J. Math. 11 (1967), 310-325.

24. E. Spanier, Algebraic topology, McGraw-Hill, New York, 1966.

25. D. Sullivan, Genetics of homotopy theory and the Adams conjecture, Ann. of Math. (2) 100 (1974), 1-79.

26. , Geometric topology, Part I, Localization, periodicity, and Galois symmetry, Mimeographed notes, M. I. T., 1970.

27. J. Wolf, The cohomology of homogeneous spaces, Amer. J. Math. 99 (1974), 312-340.

Department of Mathematics, State University of New York at Albany, Albany, New YORK 12222 